\title{
Olive oil exhibits osteoprotection in ovariectomized rats without estrogenic effects
}

\author{
XIAOHUA ZHENG ${ }^{1}$, HUIJUAN HUANG ${ }^{1},{\text { XIAOBING } \text { ZHENG }^{2} \text { and BAOHENG LI }}^{1}$ \\ ${ }^{1}$ Department of Gynecology and Obstetrics, Fuzhou General Hospital of Nanjing Command PLA, Fuzhou, Fujian 350025; \\ ${ }^{2}$ Department of Neurosurgery, The First Affiliated Hospital, Fujian Medical University, Fuzhou, Fujian 350005, P.R. China
}

Received December 23, 2014; Accepted February 11, 2016

DOI: $10.3892 / \mathrm{etm} .2016 .3138$

\begin{abstract}
The present study was designed to evaluate the effect of olive oil on bone and uterus in ovariectomized rats. A total of 34 surgically ovariectomized or sham-operated virgin Sprague-Dawley rats were divided into four groups: i) Sham-operated control rats (sham group); ii) Ovariectomized rats (OVX group); iii) Olive oil-supplemented ovariectomized rats (olive group); and iv) Diethylstilbestrol-supplemented ovariectomized rats ( $\mathrm{E}_{2}$ group). At 12 weeks following left ventricular blood sacrificed to detect plasma estradiol $\left(\mathrm{E}_{2}\right)$, interleukin-1 $\beta$ (IL-1 $\beta$ ) and IL-6 levels. Bone mineral density (BMD) of the lumbar spine was evaluated using dual-energy $\mathrm{X}$-ray absorptiometry, and the left femur proximal 1/3 slices were observed using transmission electron microscopy. Uterine wet weight and the uterus index (ratio of uterine wet weight and body weight) were compared, and the uterine endometrium was observed using a light microscope. In the OVX group, serum $\mathrm{E}_{2}$ was significantly lower and IL-1 $\beta$ and IL-6 levels were significantly higher compared with the sham group. By contrast, serum $\mathrm{E}_{2}$ levels increased and IL-1 $\beta$ levels decreased in the olive group, but showed no significant difference compared with the sham group. The lumbar spine BMD in the olive group was increased compared with OVX group. Electron microscopy revealed sparse collagen fibers in the OVX group, with decreased density and multi-cavity, showing pathological features of osteoporosis. By contrast, the situation was improved in the $\mathrm{E}_{2}$ and olive groups, in which organelles such as the rough endoplasmic reticulum, mitochondria and Golgi apparatus were visible and active. Compared with the sham group rats, the uterine wet weight and uterine index decreased in the OVX and olive groups; however, no statistically significant difference was observed in the $\mathrm{E}_{2}$ group.
\end{abstract}

Correspondence to: Mrs. Huijuan Huang, Department of Gynecology and Obstetrics, Fuzhou General Hospital of Nanjing Command PLA, 156 Xi Er-Huan North Road, Fuzhou, Fujian 350025, P.R. China

E-mail: 450523103@qq.com

Key words: gynecological malignancies, hormone replacement therapy, olive oil, postmenopausal osteoporosis, phytoestrogens
Furthermore, endometrial hyperplasia was not observed in the olive group, which were apparently different from $\mathrm{E}_{2}$ group. The present results suggest that olive oil can effectively reduce bone loss in ovariectomized rats, and with no or only mild effects on the uterine endothelium.

\section{Introduction}

Hormone replacement therapy (HRT) may exert beneficial effects in the bone of patients with postmenopausal osteoporosis; however, the chronic use of HRT has been associated with several risk factors (1). This is particularly apparent for hormone-dependent gynecological malignancies, and the appropriate usage of HRT has become a highly controversial issue $(1,2)$. Therefore, there is currently an urgent requirement for non-estrogenic, bone protective compounds. Phytoestrogens, which have similar although milder effects to those of estradiol-17 $\beta$ benzoate, are involved in the maintenance of bone mass in the post-menopausal period for their putative function as osteoprotective agents (3). Numerous traditional Chinese medicine (TCM) compounds, particularly kidney-beneficial herbal medicines such as Zuogui Pill, Rehmannia glutinosa, Ferula, in addition to various extracts from food or TCM herbs such as isoflavones (e.g. daidzein), have been suggested to prevent ovariectomy-induced bone loss and other antiosteoporotic effects without influencing hormones such as estrogen (3-6). Phytoestrogenic isoflavones and their derivatives extracted from soy have been shown to increase bone density in postmenopausal women following high dietary intake (3-7).

The human diet may contain the phytochemicals possessing antioxidant and anti-inflammatory properties, and antioxidant nutrients may enhance bone formation and reduce the production of free radicals that contribute to bone resorption $(8,9)$. Furthermore, the bone-protective effects of estrogen may involve the suppression of inflammatory cytokines such as interleukin-1 $\beta$ (IL-1 $\beta$ ), IL-6 and tumor necrosis factor- $\alpha$ (TNF- $\alpha$ ) (10). The lowest incidence of osteoporosis has been reported in the Mediterranean area within Europe $(11,12)$, due to the so-called Mediterranean diet, which reportedly exerts a beneficial effect on gynecological malignancies, such as breast and endometrial cancer $(13,14)$. Olive oil is one of the three most important and characteristic components of the Mediterranean diet, which contains a series of phenolic 
minor compounds such as hydroxytyrosol and oleuropein, and is able to scavenge superoxide radicals and inhibit neutrophil respiratory burst (15). The other two primary components of the Mediterranean diet are fruits and vegetables, and nuts and cereals.

To date, the oil extracts of numerous plant species have been demonstrated to possess similar efficacy to $17 \beta$-estradiol in suppressing bone loss due to ovariectomy and to have a marked effect in anti-osteoporosis, for example of Korean safflower seed oil, virgin coconut oil and garlic oil $(16,17)$. Furthermore, olive oil and its major phenolic compounds may exert a marked effect in osteoporosis. A prior study by Puel et al demonstrated that oleuropein and extra virgin olive oil were able to elicit protective effects on bone loss in a model of ovariectomy associated with inflammation, probably by modulating variables of inflammation, such as $\alpha$-1-acid glycoprotein (18). In another study, OVX rats were administered oleuropein (a compound extracted from olive leaf) at 2.5, 5, 10 or $15 \mathrm{mg} / \mathrm{kg}$ body weight per day for 100 days, and plasma fibrinogen concentration $(\mathrm{g} / \mathrm{l})$ was found to be significantly higher in the OVX group compared with the SH group (19). A further increase was observed after inflammation in estrogen-deficient animals, while this marker for inflammation remained unchanged in intact rats $(12,20,21)$. This pattern was partially improved by polyphenol supplementation, as fibrinogen was restored to values measured in OVX animals without talc administration (22). In this case, talc was used to stimulate and induce inflammation (22). In addition, an extra virgin olive oil total polyphenolic fraction (TPF) was isolated and its effect on the bone loss attenuation was investigated, the result showed that the TPF only slightly induced the uterine wet weight $(\mathrm{P}=0.058)(23)$. Garia-Villalba et al results suggest that postmenopausal women could be a target population for the intake of olive phenolics in order to prevent age-related and oxidative stress-related processes such as osteoporosis (23).

Consequently, the aim of the present study was to investigate the effects of olive oil supplementation in OVX rats modelling post-menopausal osteoporosis. Furthermore, olive oil was compared with $\mathrm{E}_{2}$ to determine whether olive oil has functions at the uterine tissue level in a manner similar to $17 \beta$-estradiol.

\section{Materials and methods}

Animals and treatments. A total of 34 female Sprague-Dawley rats (weight, $190 \pm 20 \mathrm{~g}$ ) were provided by the Division of Comparative Medicine of Fuzhou General Hospital of Nanjing Command PLA [permission no. SCXK (2012-0001); Fuzhou, China] were used for the present experiments. All rats were maintained in individual cages in a temperature-controlled room $\left(21^{\circ} \mathrm{C}\right)$ with a 12 -h light/dark cycle with free access to water. The rats were fed a diet constructed in the laboratory according to the normal nutritional dietary requirement $(59 \%$ carbohydrates, $7 \%$ fat, $21 \%$ protein and $13 \%$ minerals and ash). The protocol was approved by the Ethics committee of Fuzhou General Hospital of Nanjing Command PLA and was conducted in accordance with current legislation on animal experiments.

All rats were sham-operated or surgically ovariectomized under anesthesia using chloral hydrate $(4 \mathrm{ml} / \mathrm{kg}$; Fluka
Chemie AG, Buchs, Switzerland) in $80 \mathrm{~g} / 1$ saline solution $(9 \mathrm{~g} \mathrm{NaCl} / 1)$, and were randomly allocated into four groups: i) Sham-operated control rats (sham group, $n=8$ ); ii) Ovariectomized rats (OVX group, $n=9)$; iii) Diethylstilbe strol-supplemented ovariectomized rats $\left(\mathrm{E}_{2}\right.$ group, $\left.\mathrm{n}=8\right)$; and iv) olive oil-supplemented ovariectomized rats (olive group, $\mathrm{n}=9$ ). All rats were orally administered with their allocated $\operatorname{diet}(1 \mathrm{ml} / 100 \mathrm{~g})$ by oral tubal feeding for 12 weeks. Sham and OVX groups were administered daily with saline. $\mathrm{E}_{2}$ group mice received a solution of diethylstilbestrol $(0.0033 \mathrm{mg}$; Zhunzi H34021250; batch number: 20100920; Hefei Lifeon Pharmaceutical Co., Ltd., Hefei, China). Olive group mice received Terra Creta Kolymvari Chania Cretei Extra Virgin Olive Oil (Terra Creta SA, Chania, Crete, Greece), with the following component values: Acidity, $<0.5 \%$; fatty acid composition, $79 \%$ monounsaturates and $7 \%$ polyunsaturates; and trans fatty acid C18 composition, $1 \mathrm{~T} \leq 0.05(\mathrm{C} 18: 2 \mathrm{~T}+\mathrm{C} 18: 3 \mathrm{~T}$; integrated value $\leq 0.05)$. All administered substances were gavaged at $1 \mathrm{ml} / 100 \mathrm{~g}$ once daily for 12 consecutive weeks. Body mass was weighed once a week to adjust the dosage. To prevent hyperphagia associated with ovariectomy, the OVX rats were pair-fed to the mean intake of those in the sham group. At the end of the experiments, animals were sacrificed using ether (Shanghai Lianshi Chemical Reagent Co., Ltd., Shanghai, China) inhalation and exsanguination.

Serum analyses. Blood samples were centrifuged $(2,500 \mathrm{x} g$ for $20 \mathrm{~min}$ ) and the was serum stored at $-20^{\circ} \mathrm{C}$ for further analysis. Serum $\mathrm{E}_{2}$ levels were determined by radioimmunoassay kit (Fu Sheng Industrial Co., Ltd., Shanghai, China), while serum IL-1 $\beta$ and IL-6 levels was measured using an ELISA (Fu Sheng Industrial Co., Ltd.) double antibody sandwich method. The clinical laboratory of Fuzhou General Hospital was entrusted to complete all of the above test indexes according to the specific measurement of their indicators by double-blind testing.

Bone mineral density (BMD) measurements. The BMD of the lumbar spine was measured using a Discovery dual-energy X-ray absorptiometer (DXA) (S/N 806409) bone densitometer (Hologic, Inc., Bedford, MA, USA), equipped with appropriate software for bone density assessment in small laboratory animals. Unit: $\left(\mathrm{g} / \mathrm{cm}^{2}\right)$. The intra-assay and inter-assay coefficients of variability for lumbar spine assays were 0.22 and $0.24 \%$ respectively.

Femoral head visualization. Two left femoral heads of the proximal femur were selected from each group rat, cut into 1x1 $1 \mathrm{~mm}$ pieces and washed in phosphate-buffered saline (PBS) (Fuzhou Maixin Bio, Co., Ltd., Fuzhou, China). The samples were then placed in $2.5 \%$ glutaric aldehyde solution (Sinopharm Chemical Reagent Co., Ltd., Shanghai, China) for $3 \mathrm{~h}$ at $4^{\circ} \mathrm{C}$, decalcified in $5 \%$ ethylenediaminetetraacetic acid (EDTA) (Fuzhou Maixin Bio, Co., Ltd.) for three weeks by changing EDTA solution every week. Subsequently, the samples were sent to the Department of Pathology to be fixed in $1 \%$ osmium tetroxide, embedded and cut into thin sections with Epon812 (both purchased from SPI Supplies, West Chester, PA, USA), double-stained using uranyl acetate (China National Nuclear Corporation, Beijing, China) and 
Table I. Serum $\mathrm{E}_{2}$ measured in groups (mean \pm standard deviation).

\begin{tabular}{|c|c|c|c|c|}
\hline Group & $\mathrm{n}$ & $\mathrm{E}_{2}(\mathrm{ng} / \mathrm{ml})$ & $\mathrm{P}^{\mathrm{a}}$ & $\mathrm{P}^{\mathrm{b}}$ \\
\hline Sham & 8 & $66.765 \pm 4.394$ & - & 0.000 \\
\hline OVX & 9 & $30.087 \pm 5.504$ & 0.000 & - \\
\hline $\mathrm{E}_{2}$ & 8 & $45.226 \pm 4.745$ & 0.005 & 0.038 \\
\hline Olive & 9 & $54.452 \pm 4.774$ & 0.087 & 0.001 \\
\hline
\end{tabular}

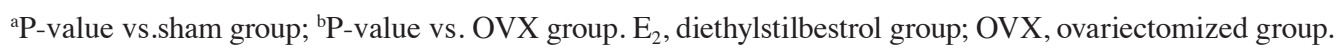

Table II. Serum IL-1 $\beta$ measured in groups (mean \pm standard deviation).

\begin{tabular}{|c|c|c|c|c|}
\hline Group & $\mathrm{n}$ & IL-1 $\beta(\mathrm{pg} / \mathrm{ml})$ & $\mathrm{P}^{\mathrm{a}}$ & $\mathrm{P}^{\mathrm{b}}$ \\
\hline Sham & 8 & $83.764 \pm 6.508$ & - & 0.012 \\
\hline OVX & 9 & $124.273 \pm 14.470$ & 0.012 & - \\
\hline $\mathrm{E}_{2}$ & 8 & $91.730 \pm 9.816$ & 0.612 & 0.039 \\
\hline Olive & 9 & $93.417 \pm 9.209$ & 0.527 & 0.044 \\
\hline
\end{tabular}

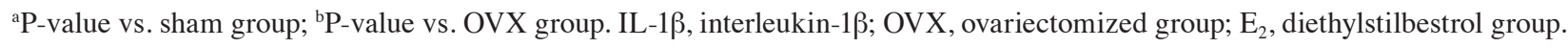

Table III. Serum IL-6 measured in groups (mean \pm standard deviation).

\begin{tabular}{lccrc}
\hline Group & $\mathrm{n}$ & IL-6 (pg/ml) & $\mathrm{P}^{\mathrm{a}}$ & $\mathrm{P}^{\mathrm{b}}$ \\
\hline Sham & 8 & $111.433 \pm 16.574$ & - & 0.001 \\
OVX & 9 & $226.700 \pm 23.731$ & 0.001 & - \\
$\mathrm{E}_{2}$ & 8 & $126.885 \pm 18.996$ & 0.622 & 0.002 \\
Olive & 9 & $176.254 \pm 23.447$ & 0.040 & 0.095 \\
\hline
\end{tabular}

aP-value vs. sham group; ${ }^{b} \mathrm{P}-\mathrm{value}$ vs. OVX group. IL-6, interleukin-6; OVX, ovariectomized group; $\mathrm{E}_{2}$, diethylstilbestrol group.

lead citrate (Sinopharm Chemical Reagent Co., Ltd.), finally observed using transmission electron microscope (EM208S; Philips, Amsterdam, Netherlands).

Uterine endothelium visualization. Samples of uterine tissues were fixed, embedded in paraffin and cut into serial cross sections. The skin was cut vertically in the median of the lower abdomen and the peritoneum was opened into the abdominal cavity. The ' $\mathrm{Y}$ ' type uterus was extracted and adipose tissue removed. Wet weight was recorded and the uterus index calculated (uterine wet weight/body weight). Then, two embedded rat uteri were selected from each group, and made into paraffin sections. The extent of endometrial hyperplasy was observed under a light microscope [2506; Motic (Xiamen) Electric Group Co., Ltd., Xiamen, China] and photographed using a microscope with a digital camera (BX51; Olympus Corporation, Tokyo, Japan).

Statistical analysis. Data are expressed as the mean \pm standard deviation. Statistical significance for data was determined using one-way analysis of variance with post-hoc test, significance calculated using a Least Significant Difference multiple range-test to find inter-group significance. $\mathrm{P}<0.05$ was considered to indicate a statistically significant difference.

\section{Results}

Comparison of serum $E_{2}$ levels among groups. By analysis of variance indicated statistically significant differences in $\mathrm{E}_{2}$ expression among groups $(\mathrm{F}=9.934$; overall significance, $\mathrm{P}=0.000)$. Serum $\mathrm{E}_{2}$ values in the olive group were not significantly different compared with the sham group $(\mathrm{P}>0.05)$; however, they were significantly increased compared with the OVX group ( $\mathrm{P}=0.001)$, as shown in Table $\mathrm{I}$.

Comparison of serum IL-1 $\beta$ levels among groups. Serum IL-1 $\beta$ concentration was similar in the sham, $\mathrm{E}_{2}$ and olive groups $(\mathrm{P}>0.05)$. This marker was increased in the OVX animals, but was mitigated in the olive and $\mathrm{E}_{2}$ group rats (Table II).

Comparison of serum IL-6 levels among groups. In OVX group, IL-6 levels were significantly higher than Sham group and $E_{2}$ group, the difference was statistically significant $(\mathrm{P}<0.01)$, but no statistical significance with Olive group 
Table IV. BMD of the lumbar spine among groups (mean \pm standard deviation).

\begin{tabular}{lcccc}
\hline Group & $\mathrm{n}$ & $\mathrm{BMD}\left(\mathrm{g} / \mathrm{cm}^{2}\right)$ & $\mathrm{P}^{\mathrm{a}}$ & $\mathrm{P}^{\mathrm{b}}$ \\
\hline Sham & 8 & $0.2476 \pm 0.0049$ & - & 0.004 \\
OVX & 9 & $0.2262 \pm 0.0049$ & 0.004 & - \\
$\mathrm{E}_{2}$ & 8 & $0.2291 \pm 0.0053$ & 0.015 & 0.679 \\
Olive & 9 & $0.2431 \pm 0.0046$ & 0.521 & 0.018 \\
\hline
\end{tabular}

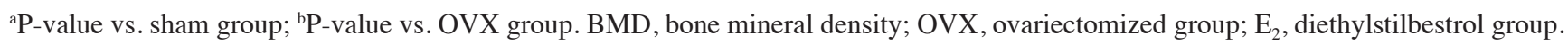

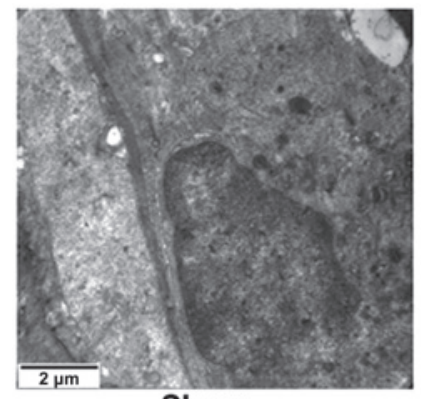

Sham

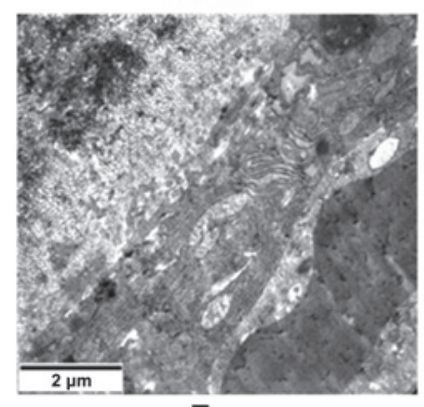

$E_{2}$

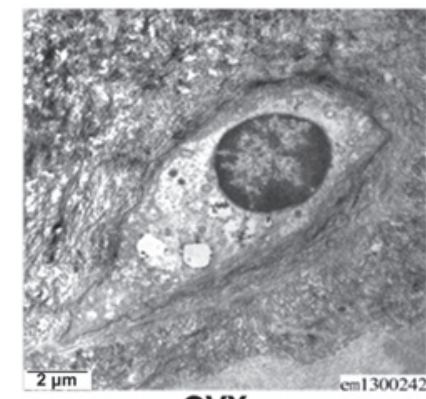

OVX

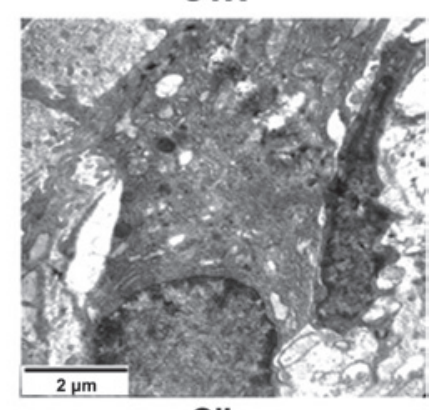

Olive
Figure 1. Collagen fibers and organelles of the femoral head under transmission electron microscope imaging in the four groups. OVX, ovariectomized group; $\mathrm{E}_{2}$, diethylstilbestrol group.

( $P>0.05)$. There was not statistically significant in $\mathrm{E}_{2}$ group compared with Sham group ( $\mathrm{P}>0.05)$, as shown in Table III.

Comparison of absolutes values $\left(\mathrm{g} / \mathrm{cm}^{2}\right)$ of BMD of the lumbar spine among groups. Lumbar spine BMD in the olive group was significantly higher compared with the OVX group, but showed no significant difference compared with the sham group. However, lumbar spine BMD was not significantly different between the $\mathrm{E}_{2}$ and OVX groups ( $\left.\mathrm{P}>0.05\right)$, as shown in Table IV.

Femoral head under transmission electron microscopy. Collagen fibers in the sham group showed light and dark stripes, closely and neatly arranged. In addition, osteoblasts and numerous Golgi apparatus, endoplasmic reticula and other organelles could be seen, as shown in Fig. 1.

Collagen fibers in the OVX group were sparse, exhibited bending and breaking, and numerous voids are visible, also osteoclast were clearly visible, presenting pathological features of osteoporosis, as shown in Fig. 1.

The arrangement of collagen fibers in the $\mathrm{E}_{2}$ and olive groups improved compared with OVX group, with organelles

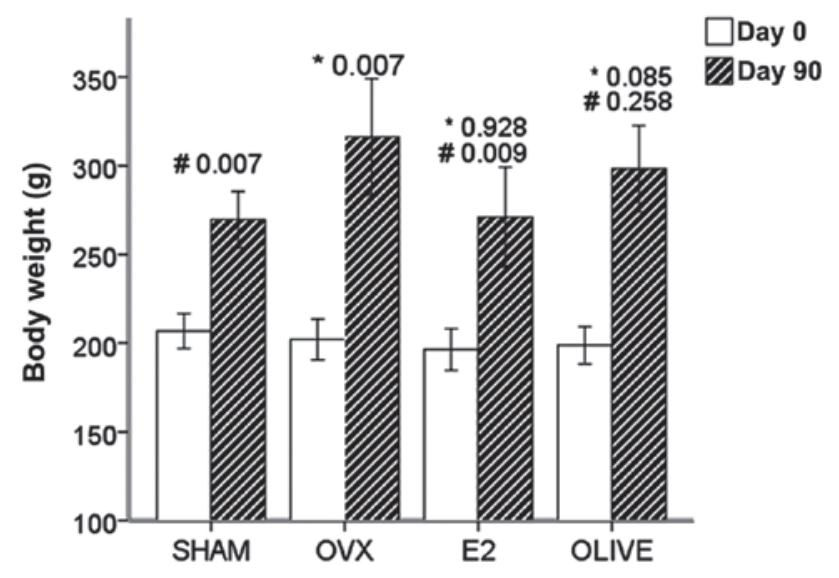

Figure 2. Body weight pre-treatment and post-treatment among four groups. Body weight after 90 days: "P-value vs. sham group; "P-value vs. OVX group. OVX, ovariectomized group; $\mathrm{E}_{2}$, diethylstilbestrol group.

such as the rough endoplasmic reticulum, mitochondria and Golgi apparatus being visible and active, as shown in Fig. 1.

Body weight pre-treatment and post-treatment. None of the groups exhibited a significant difference in mean initial body weight $(\mathrm{P}>0.05)$. At 90 days after operation, weights of OVX group rats were increased by $57.16 \%$, which was increased compared with the sham group (30.95\%). The two groups showed a significant difference in body weight after 90 days (sham, 269.63 $\pm 6.70 \mathrm{~g}$ vs. OVX, 316.33 $\pm 14.20 \mathrm{~g}, \mathrm{P}=0.007$ ). Weights of $\mathrm{E}_{2}$ group rats showed no significant difference compared with the sham group rats, but were reduced compared with the OVX group rats. Weights of the olive group rats did not significantly differ from those of the OVX and sham groups $(\mathrm{P}>0.05)$ (Fig. 2).

Uterine weight. Uterine weights and uterine index values in the OVX and olive group rats were significantly decreased compared with the sham group rats $(\mathrm{P}<0.001)$. Treatment of $\mathrm{E}_{2}$ group significantly increased uterine weights, significantly different with OVX group, but had no significant difference with sham group $(\mathrm{P}>0.05)$. The $\mathrm{E}_{2}$ group exhibited no significant difference compared with the OVX group $(\mathrm{P}>0.05)$ (Table V).

Comparison of histomorphometric variables of uterine among groups. HE staining and visualization using light microscopy at low magnification (magnification, $x 40$ ) revealed that the olive and OVX groups showed thin endometria, with no 
Table V. Uterine weight and uterine index among groups (mean \pm standard deviation).

\begin{tabular}{lcccc}
\hline Group & Uterine weight $(\mathrm{mg})$ & Uterine index $(\mathrm{mg} / \mathrm{g})$ & $\mathrm{P}^{\mathrm{a}}$ & $\mathrm{P}^{\mathrm{b}}$ \\
\hline Sham & $674.05 \pm 68.40$ & $2.485 \pm 0.227$ & - & 0.000 \\
OVX & $116.71 \pm 15.54$ & $0.358 \pm 0.035$ & 0.000 & - \\
E $_{2}$ & $539.61 \pm 58.15$ & $2.061 \pm 0.299$ & 0.118 & 0.000 \\
Olive & $233.69 \pm 22.17$ & $0.785 \pm 0.073$ & 0.000 & 0.097
\end{tabular}

${ }^{\text {ap}} \mathrm{P}$-value vs. sham group; ${ }^{\mathrm{b}} \mathrm{P}$-value vs. OVX group. OVX, ovariectomized group; $\mathrm{E}_{2}$, diethylstilbestrol group.

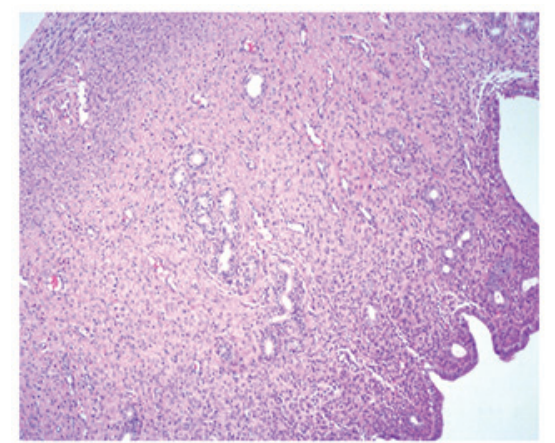

Sham

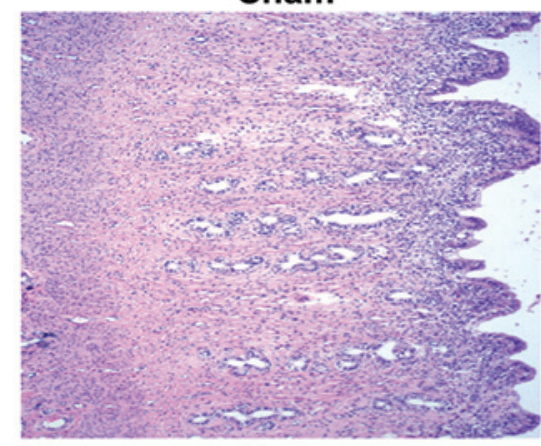

$E_{2}$

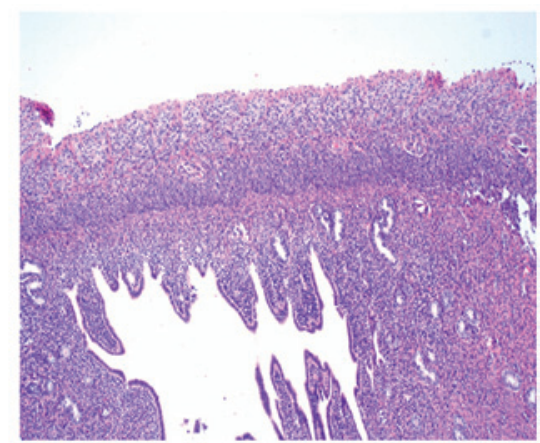

ovx

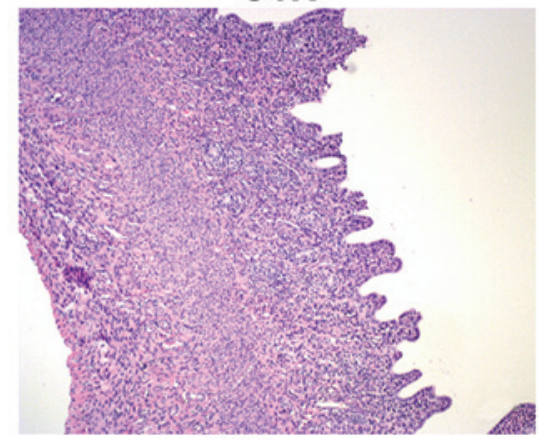

Olive

Figure 3. Morphology of the endometrium among the four groups. OVX, ovariectomized group; $\mathrm{E}_{2}$, diethylstilbestrol group.

proliferation. However, in the $\mathrm{E}_{2}$ group the columnar epithelial thickened, the number of glands increased, with evident hyperplasia (Fig. 3).

\section{Discussion}

Ovarian, cervical and endometrial cancers are common gynecological malignancies, whose incidence are increasing (24-26). This is particularly evident in the case of ovarian cancer, which poses a serious threat to female health (25). With the continuous development of medical diagnosis and treatment technology, the cure rate and disease-free survival of patients has improved significantly (27). However, radical surgery and adjuvant chemotherapy-induced menopause syndrome are becoming serious factors affecting the quality of life of patients (28), particularly osteoporosis, whose risk is significantly higher than natural menopause, and the problems caused by its fracture in postmenopausal women has been described as a 'silent killer' (29). Hormone replacement therapy (HRT) has been publicly recognized as the first choice prevention and treatment for postmenopausal osteoporosis, and the majority of obstetrician-gynecologists are aware of the osteoporosis problem. However, non-HRT users are often screened, and in a recent survey $69.4 \%$ of gynecologists speculated that HRT could increase the risk of breast cancer, and $52.9 \%$ speculated that HRT may increase the risk of endometrial cancer (30). The appropriate use of HRT for the treatment of osteoporosis and postoperative gynecological malignancies has become a highly controversial issue, particularly in the case of hormone-dependent gynecological malignancies (31). Therefore, there is a requirement for treatments for osteoporosis that have fewer undesirable side effects on the female reproductive system as estradiol-17 $\beta$ benzoate.

The present results show that the administration of olive oil to ovariectomized rats resulted in increased serum levels of $\mathrm{E}_{2}$ compared with the OVX group, but with no statistically significant difference compared with the sham group. The serum IL-1 $\beta$ level in the olive group rats showed no statistically significant difference compared with the sham and $E_{2}$ groups. The serum IL-6 level in the olive group rats did not differ significantly compared with the OVX group. These results indicate that olive oil supplementation can result in 
increased serum $E_{2}$ and decreased IL-1 $\beta$ levels. By contrast, diethylstilbestrol supplementation of the ovariectomized rats increased serum $\mathrm{E}_{2}$ and decrease IL-1 $\beta$ and IL-6 levels.

Ovariectomy induced a significant reduction in total BMD (OVX, $0.2262 \pm 0.0049$ vs. sham, $0.2476 \pm 0.0049 \mathrm{~g} / \mathrm{cm}^{2}$; $\mathrm{P}=0.004)$. However, olive oil reduced this bone loss and showed no significant difference compared with the $\mathrm{E}_{2}$ group (olive, $0.2431 \pm 0.0046$ vs. $\mathrm{E}_{2} 0.2291 \pm 0.0053 \mathrm{~g} / \mathrm{cm}^{2} ; \mathrm{P}=0.053$ ). The arrangement of collagen fibers in the olive group were more tightly packed and regular compared with the OVX group, while organelles such as the rough endoplasmic reticulum, mitochondria and Golgi apparatus were visible and active, indicating micro proliferation of bone.

Estrogen deficiency induces a mild increase in the production of proinflammatory cytokines, such as IL- $1 \beta$, IL- 6 , and TNF- $\alpha$, systemically and locally, which promotes osteoclastogenesis in the bone marrow $(32,33)$. IL-1 $\beta$ and IL-6 are proinflammatory cytokines involved in bone turnover. IL- $1 \beta$ is a potent stimulant of bone resorption, acting directly on osteoclasts and osteoclast precursors (34). Numerous studies have investigated the associations between IL-1 $\beta$ gene polymorphisms and susceptibility to osteoporosis and BMD in various populations $(35,36)$. A prior study enrolled 226 postmenopausal women with a diagnosed BMD T-score $<-2.5$ standard deviation (SD) and 224 postmenopausal women with a BMD T-score $>-2.5 \mathrm{SD}$ to investigate the associations between cytokine gene polymorphisms (IL-1 $\beta$, IL-2 and IL-6) and BMD values (37). The result suggested that among the women with T-scores $<-2.5 \mathrm{SD}$, the BMD values were significantly lower in the carriers of the IL-6 GG genotype compared with those with the CC and GC genotypes. Furthermore, there was a statistically significant association between the IL- $6-174 \mathrm{G} / \mathrm{C}$ polymorphism and osteoporosis in postmenopausal women (37). As the immune system plays such a critical role in the pathophysiology of postmenopausal osteoporosis, cytokines have the ability to influence each other; just as a mild increase in the production of proinflammatory cytokines would be found in a patient with osteoporosis, an increase in proinflammatory cytokines may exacerbate osteoporosis (37). Terauchi has suggested that counteracting these interactions could be a novel strategy for osteoporosis treatment (38). Puel et al showed that the daily consumption of the major phenolic compounds in olive oil for 84 days modulated bone loss in an ovariectomy/inflammation experimental model, concluding that polyphenol consumption of olive oil may be associated with the mitigation of bone loss (22).

The aforementioned results suggest that olive oil is able to increase $\mathrm{E}_{2}$ levels, and had positive effects on proinflammatory cytokines IL-1 $\beta$ and bone loss, as indicated by the collagen fibers of femoral, indicating that olive oil supplementation in OVX-rats attenuated ovariectomy-induced osteoporosis. A possible reason for this improvement in bone loss could be attributed to the elevated levels of $E_{2}$ and the high content of monounsaturated fatty acid in olive oil, which has been reported to affect BMD (39). However, as supplementation with diethylstilbestrol was also able to increase serum $\mathrm{E}_{2}$ and decrease IL-1 $\beta$ and IL-6 levels in the ovariectomized rats, the estrogenic effects were more marked compared with olive oil. It is possible that the total quantity of olive oil administered in this experiment were insufficient to equal the effects of diethylstilbestrol.

The body weights of the $\mathrm{E}_{2}$ group rats were similar to the sham group rats, but reduced compared with the OVX group rats. Body weights of the olive group rats showed no significant difference compared with sham group rats $(P>0.05)$, suggesting that olive oil may not share the positive effect of controlling body weight increase induced by ovariectomy as exhibited by estrogen supplement. A diet rich in unsaturated fatty acids from olive oil reduces waist girth, a significant indicator of metabolic disease, in addition to reducing body mass index (40). In addition, a study supports the hypothesis that extra virgin olive oil phenols protect low density lipoproteins in the plasma against oxidation (41).

Uterine wet weight and uterine index values of the olive group rats decreased compared with sham group $(\mathrm{P}<0.01)$, but showed no significant difference compared with the OVX group $(\mathrm{P}>0.05)$. By contrast, the uterine weight and uterine index of the $\mathrm{E}_{2}$ group rats significantly increased compared with the OVX group $(\mathrm{P}<0.01)$, and the uterine index was not statistically significant compared with the sham group. Furthermore, the endometria in the olive group were atrophic, thinner and the number of glands was reduced, as imaged using a light microscope. The endometria exhibited hyperplasia in the $\mathrm{E}_{2}$ group, and were significantly different compared with the olive and OVX groups. These results suggest that olive oil had no significant or only a mild effect on the uterus, indicating that it may be safe to use as a form of hormone replacement therapy in treating female genital mutilation. The results may be similar to Keiler AM and colleagues' study, in which female Lewis rats ovariectomized and fed a diet enriched with a total phenolic extract of extra virgin olive oil in a concentration of $800 \mathrm{mg} / \mathrm{kg}$ diet for 12 weeks (42). The results showed that oleocanthal, a compound of the polyphenolic fraction, showed a higher relative estrogen receptor binding affinity to the estrogen receptor $\alpha(E R \alpha)$ compared to the ER $\beta$. While the total polyphenolic fraction (TFP) only mildly increased the uterine wet weight ( $490.7 \pm 53.7$ vs. $432.7 \pm 23 \mathrm{mg} / \mathrm{kg}$ body weight; $\mathrm{P}=0.058$ ), it regulated estrogen response genes in the uterus (progesterone receptor, antigen identified by monoclonal antibody Ki67, complement C3). These results suggested that the administration of extra virgin olive oil polyphenols regulated uterine estrogen response marker genes in an $\mathrm{E}_{2}$-agonistic manner (42).

Olive oil may be rich in phytoestrogens, similar to other plants or their ingredient extracts. Traditional Chinese medicinal herbs such as Rehmannia glutinosa and Ferula, or oils extracted from various plant species, which have similar though milder effects to that of estradiol-17 $\beta$ benzoate, may be associated with the maintenance of bone mass in the post-menopausal period due to their putative function as osteoprotective agents (42).

Estrogen exerts certain effects by binding to different estrogen receptors, including ER $\alpha$ and $E R \beta$. ER $\alpha$ is predominant in a number of tissues and is primarily involved in the reproductive system, whereas ER $\beta$ is expressed in numerous tissues including bone (34). ER $\alpha$ and ER $\beta$ may be present in rat and human osteoblasts in cortical and trabecular compartments of bone $(36,37)$, and a number of phytoestrogens, 
including genistein and coumestrol, show a higher affinity to $\operatorname{ER} \beta(20)$.

A possible explanation for olive oil exhibiting bone protective effects but no estrogenic effects may be its phytoestrogen effect; olive oil is structurally related to $17 \beta$-estradiol, but has much stronger affinity for ER $\beta$ than $17 \beta$-estradiol. As Annekathrin Martina Keiler do has proved the polyphenols impact on estrogen response genes because of the aromatic ring structurally related to $17 \beta$-estradiol binding to estrogen receptors. Seidlova-Wuttke et al previously showed that $\beta$-ecdysone has an antiosteoporotic effect which does not involve the activation of ER in ovariectomized rats. Thus, olive oil may a exert a similar bone protective effect, with no associated estrogenic effects (21).

Furthermore, olive oil also has anti-cancer effect as there has been reported that olive oil contains a number of compounds which by their antioxidative action reduce the risk of cell damage and their consequential uncontrolled growth and division (43). Therefore, olive oil may be more suitable to the treatment of osteoporosis associated with postoperative gynecological malignancies, displaying desirable estrogenic effects but with fewer undesirable side effects.

In conclusion, the present study demonstrates the beneficial effects of olive oil on osteoporosis via serum proinflammatory cytokines and lumbar spine BMD. However, further research is required in order to determine the mechanism of its effects, possibly using an ER-ligand binding assay or phytoestrogen.

\section{Acknowledgements}

This study was supported by the Nanjing Command Medical Science and Technology Innovation Project (no. 11MA111).

\section{References}

1. Rozenberg S, Murillo D, Gevers R and Vandromme J: Propensity of gynaecologists towards osteoporosis management and treatment. Maturitas 53: 483-488, 2006.

2. Barakat RR, Bundy BN, Spirtos NM, Bell J and Mannel RS; Gynecologic Oncology Group Study: Randomized double-blind trial of estrogen replacement therapy versus placebo in stage I or II endometrial cancer: A Gynecologic Oncology Group Study. J Clin Onco1 24: 587-592, 2006.

3. Lim DW and Kim YT: Dried Root of Rehmannia glutinosa prevents bone loss in ovariectomized rats. Molecules 18: 5804-5813,2013.

4. Liu MJ, Li Y, Pan JH, Liu H, Wang SJ, Teng JR, Zhao HY and $\mathrm{Ju} \mathrm{DH}$ : Effects of zuogui pill (see text) on Wnt signal transduction in rats with glucocorticoid-induced osteoporosis. J Tradit Chin Med 31: 98-102, 2011.

5. Palumbo C, Ferretti M, Bertoni L, Cavani F, Resca E, Casolari B, Carnevale G, Zavatti M, Montanari C, Benelli A and Zanoli P: Influence of ferutinin on bone metabolism in ovariectomized rats. I: Role in preventing osteoporosis. J Bone Miner Metab 27: 538-545, 2009.

6. Tyagi AM, Srivastava K, Sharan K, Yadav D, Maurya R and Singh D: Daidzein prevents the increase in CD $4^{+} \mathrm{CD} 28$ null T cells and B lymphopoesis in ovariectomized mice: A Key Mechanism for anti-osteoclastogenic effect. PLoS One 6: e21216, 2011.

7. Mei J, Yeung SS and Kung AW: High dietary phytoestrogen intake is associated with higher bone mineral density in postmenopausal but not premenopausal women. J Clin Endocrinol Metab 86: 5217-5221, 2001.

8. Puel C, Mardon J, Kati-Coulibaly S, Davicco MJ, Lebecque P, Obled C, Rock E, Horcajada MN, Agalias A, Shaltsounis LA and Coxam V: Black Lucques olives prevented bone loss caused by ovariectomy and talcgranulomatosis in rats. J Brit Nutri 97: 1012-1020, 2007
9. Rendina E, Hembree KD, Davis MR Marlow D, Clarke SL, Halloran BP, Lucas EA and Smith BJ: Dried plum's unique capacity to reverse bone loss and alter bone metabolism in postmenopausal osteoporosis model J PLoS One 8: e60569, 2013

10. Kim HM, An CS, Jung KY, Choo YK, Park JK and Nam SY: Rehmannia glutinosa inhibits tumour necrosis factor-alpha and interleukin-1 secretion from mouse astrocytes. Pharmacol Res 40: 171-176, 1999.

11. Kanis JA: The incidence of hip fracture in Europe. Osteoporos Int 3 (Suppl 1): S10-S15, 1993.

12. Benetou V, Orfanos P, Pettersson-Kymmer U, Bergström U, Svensson O, Johansson I, Berrino F, Tumino R, Borch KB, Lund E, et al: Mediterranean diet and incidence of hip fractures in a European cohort. Osteoporos Int 24: 1587-1598, 2013.

13. Buckland G, Travier N, Cottet V, González CA, Luján-Barroso L, Agudo A, Trichopoulou A, Lagiou P, Trichopoulos D, Peeters $\mathrm{PH}$, et al: Adherence to the mediterranean diet and risk of breast cancer in the European prospective investigation into cancer and nutrition cohort study. Int J Cancer 132: 2918-2927, 2013.

14. Dalvi TB, Canchola AJ and Horn-Ross PL: Dietary patterns, Mediterranean diet, and endometrial cancer risk. Cancer Causes Control 18: 957-966, 2007.

15. Omar SH: Oleuropein in olive and its pharmacological effects. Sci Pharm 78: 133-154, 2010

16. Mukherjee M, Das AS, Das D, Mukherjee S, Mitra S and Mitra C: Effects of garlic oil on postmenopausal osteoporosis using ovariectomized rats: Comparison with the effects of lovastatin and 17beta-estradiol. Phytother Res 20: 21-27, 2006.

17. Mukherjee M, Das AS, Mitra S and Mitra C: Prevention of bone loss by oil extract of garlic (Allium sativum Linn) in an ovariectomized rat model of osteoporosis. Phytother Res 18: 389-394, 2004.

18. Puel C, Mardon J, Agalias A, Davicco MJ, Lebecque P, Mazur A, Horcajada MN, Skaltsounis AL and Coxam V: Major phenolic compounds in olive oil modulate bone loss in an ovariectomy/inflammation experimental model. J Agric Food Chem 56: 9417-9422, 2008

19. Puel C, Mathey J, Agalias A, Kati-Coulibaly S, Mardon J, Obled C, Davicco MJ, Lebecque P, Horcajada MN, Skaltsounis AL and Coxam V: Dose-response study of effect of oleuropein, an olive oil polyphenol, in an ovariectomy/inflammation experimental model of bone loss in the rat. Clin Nutr 25: 859-868, 2006.

20. Moreira AC, Silva AM, Santos MS and Sardão VA: Phytoestrogens as alternative hormone replacement therapy in menopause: What is real, what is unknown. J Steroid Biochem Mol Biol 143: 61-71, 2014.

21. Seidlova-Wuttke D, Christel D, Kapur P, Nguyen BT, Jarry H and Wuttke W: Beta-ecdysone has bone protective but no estrogenic effects in ovariectomized rats. Phytomedicine 17: 884-889, 2010.

22. Puel C, Quintin A, Agalias A, Mathey J, Obled C, Mazur A, Davicco MJ, Lebecque P, Skaltsounis AL and Coxam V: Olive oil and its main phenolic micronutrient (oleuropein) prevent inflammation-induced bone loss in the ovariectomized rat. $\mathrm{Br}$ J Nutr 92: 119-127, 2004.

23. García-Villalba R, Larrosa M, Possemiers S, Tomás-Barberán FA and Espín JC: Bioavailability of phenolics from an oleuropein-rich olive (Olea europaea) leaf extract and its acute effect on plasma antioxidant status: Comparison between pre- and postmenopausal women. Eur J Nutr 53: 1015-1027, 2014.

24. Dietl J: Revisiting the pathogenesis of ovarian cancer: the central role of the fallopian tube. Arch Gynecol Obstet. 289: 241-246, 2014.

25. Acharya UR, Sree SV, Saba L, Molinari F, Guerriero S and Suri JS: Ovarian tumor characterization and classification using ultrasound-a new online paradigm. J Digit Imaging 26: 544-553, 2013.

26. Cormio A, Cormio G, Musicco C, Sardanelli AM, Gasparre G and Gadaleta MN: Mitochondrial changes in endometrial carcinoma: possible role in tumor diagnosis and prognosis. Oncol Rep 33: 1011-1018, 2015.

27. Poveda A, Ray-Coquard I, Romero I, Lopez-Guerrero JA, and Colombo N: Emerging treatment strategies in recurrent platinum-sensitive ovarian cancer: focus on trabectedin. Cancer Treat Rev 40: 366-375, 2014

28. Nishio K, Tanabe A, Maruoka R, Nakamura K, Takai M, Sekijima T, Tunetoh S, Terai Y and Ohmichi M: Bone mineral loss induced by anticancer treatment for gynecological malignancies in premenopausal women. Endocr Connect 2: 11-17, 2012. 
29. Chang SF, Hong CM and Yang RS: The performance of an online osteoporosis detection system a sensitivity and specificity analysis. J Clin Nurs. 23: 1803-1809, 2014.

30. Wang Y, Yang X, Li X, He X and Zhao Y: Knowledge and personal use of menopausal hormone therapy among Chinese Obstetrician-gynecologists: Results of a survey. Menopause 21: 1190-1196, 2014

31. Stavraka C,Maclaran K, Gabra H,AgarwalR, Ghaem-MaghamiS, Taylor A, Dhillo WS, Panay N and Blagden SP: A study to evaluate the cause of bone demineralization in gynecological cancer survivors. Oncologist 18: 423-429, 2013.

32. Orsal E, Halici Z, Bayir Y, Cadirci E, Bilen H, Ferah I, Aydin A, Ozkanlar S, Ayan AK, Seven B and Ozaltin S: The role of carnitine on ovariectomy and inflammation-induced osteoporosis in rats. Exp Biol Med 238: 1406-1412, 2013.

33. Brincat SD, Borg M, Camilleri G and Calleja-Agius J: The role of cytokines in postmenopausal osteoporosis. Minerva Ginecol 66 391-407, 2014.

34. Knudsen S, Harsløf T, Husted LB, Carstens M, Stenkjaer L and Langdahl BL: The effect of interleukin-1alpha polymorphisms on bone mineral density and the risk of vertebral fractures. Calcif Tissue Int 80: 21-30, 2007.

35. Kim JG, Lim KS, Ku SY, Kim SH, Choi YM and Moon SY: Relations between interleukin-1, its receptor antagonist gene polymorphism and bone mineral density in postmenopausal Korean women. J Bone Miner Metab 24: 53-57, 2006.

36. Chen HY, Chen WC, Wu MC, Tsai FJ and Lin CC: Interleukin-1beta and interleukin-1 receptor antagonist gene polymorphism in postmenopausal women: Correlation to bone mineral density and susceptibility to osteoporosis. Maturitas 44: 49-54, 2003.
37. Czerny B, Kaminski A, Kurzawski M, Kotrych D, Safranow K, Dziedziejko V, Bohatyrewicz A and Pawlik A: The association of IL-1beta, IL-2 and IL-6 gene polymorphisms with bone mineral density and osteoporosis in postmenopausal women. Eur J Obstet Gynecol Reprod Biol 149: 82-85, 2010.

38. Terauchi M: Role of the immune system in the pathophysiology of postmenopausal osteoporosis. Nihon Rinsho 69: 1215-1219, 2011 (In Japanese)

39. Saleh NK and Saleh HA: Olive oil effectively mitigates ovariectomy-induced osteoporosis in rats. BMC Complement Altern Med 11: 10, 2011.

40. Paniagua JA, Gallego de la Sacristana A, Romero I, Vidal-Puig A, Latre JM, Sanchez E, Perez-Martinez P, Lopez-Miranda J and Perez-Jimenez F: Monounsaturated fat-rich diet prevents central body fat distribution and decreases postprandial adiponectin expression induced by a carbohydrate-rich diet in insulin-resistant subjects. Diabetes Care 30: 1717-1723, 2007.

41. Leenen R, Roodenburg AJ, Vissers MN, Schuurbiers JA, van Putte KP, Wiseman SA and van de Put FH: Supplementation of plasma with olive oil phenols and extracts: Influence on LDL oxidation. J Agric Food Chem 27: 1290-1297, 2002.

42. Keiler AM,Zierau O, Bernhardt R, Scharnweber D, Lemonakis N, Termetzi A, Skaltsounis L, Vollmer G and Halabalaki M: Impact of a functionalized olive oil extract on the uterus and the bone in a model of postmenopausal osteoporosis. Eur J Nutr 53: 1073-1081, 2014.

43. Keiler AM,Zierau O, Bernhardt R, Scharnweber D, Lemonakis N, Termetzi A, Skaltsounis L, Vollmer G and Halabalaki M: Impact of a functionalized olive oil extract on the uterus and the bone in a model of postmenopausal osteoporosis. Eur J Nutr 53: 1073-1081, 2014. 